\title{
The DUNE Near Detector Complex
}

\section{Alan Bross}

Physics Opportunities in the Near DUNE Detector Hall

December $3^{\text {rd }}, 2018$ 


\section{Outline}

- Motivation and overview of the baseline facility

- Flux, event rates and beam systematics

- Physics program (Chris Marshall)

- Brief introduction to the DUNE Near detectors

- LAr (James Sinclair)

- Multi-purpose Detector (MPD) (Tanaz Mohayai)

- 3DST (Clark McGrew)

- DUNE-PRISM (Christovao Vilela)

- Near detector hall

- Conclusions and outlook

Details in the talks that follow 


\section{Why do we need near detector(s)}

CP Violation Sensitivity

\section{Primary purpose}

The significance with which CP violation, defined as $\delta C P$ not equal to zero or $\pi$, as a function of exposure in kt-MW-years, for equal running in $\mathrm{FHC}$ and $\mathrm{RHC}$ mode. True normal ordering is assumed. The width of the band corresponds to the difference in sensitivity between $v_{e}$ signal normalization uncertainty of $1 \%$ and $3 \%$ with $5 \%$ uncertainty on the $v_{\mu}$ disappearance mode.

\section{+ many topics on physics} beyond vSM (I think that is why we are here)

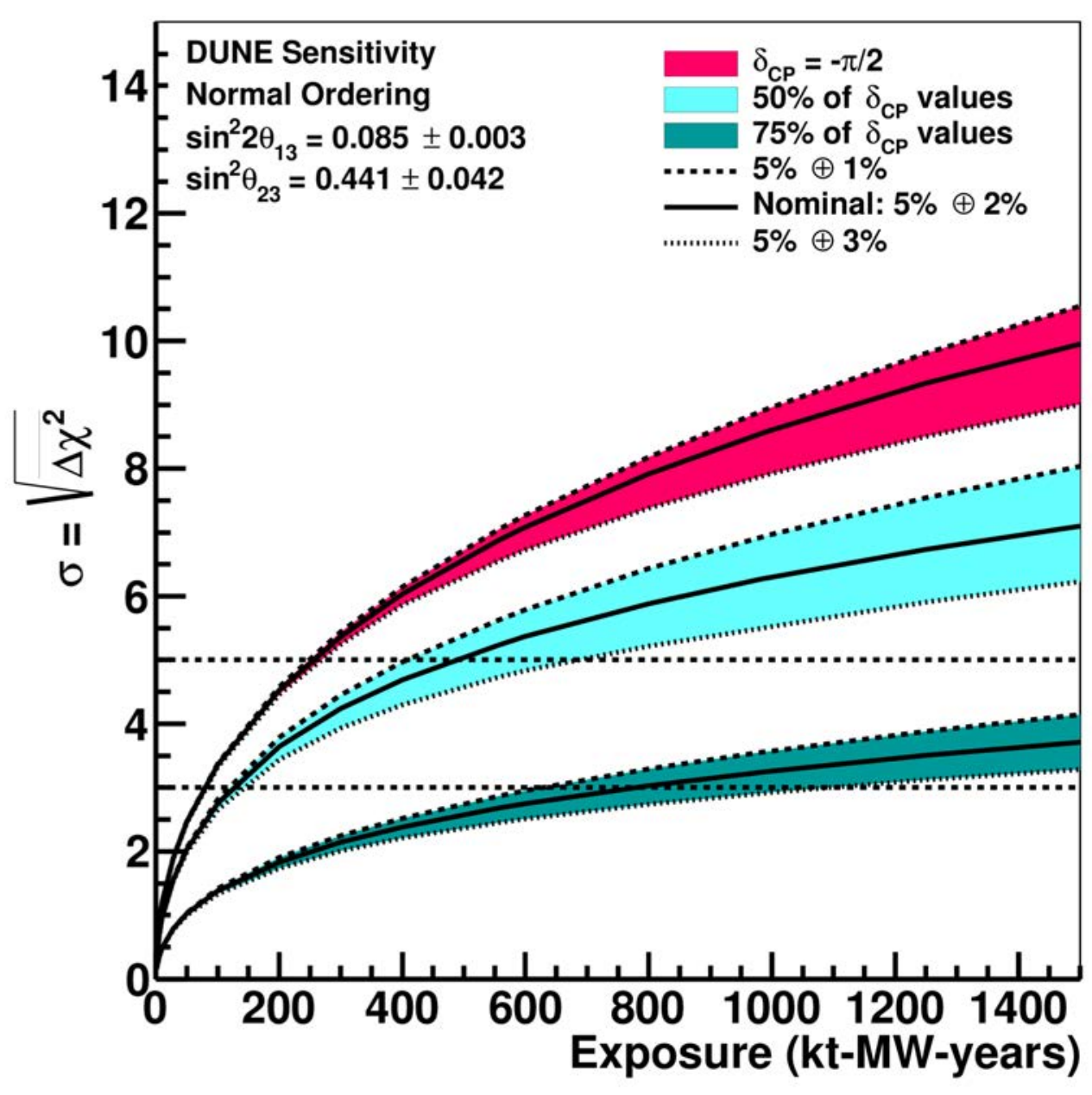




\section{Facility: Bird's-Eye View}

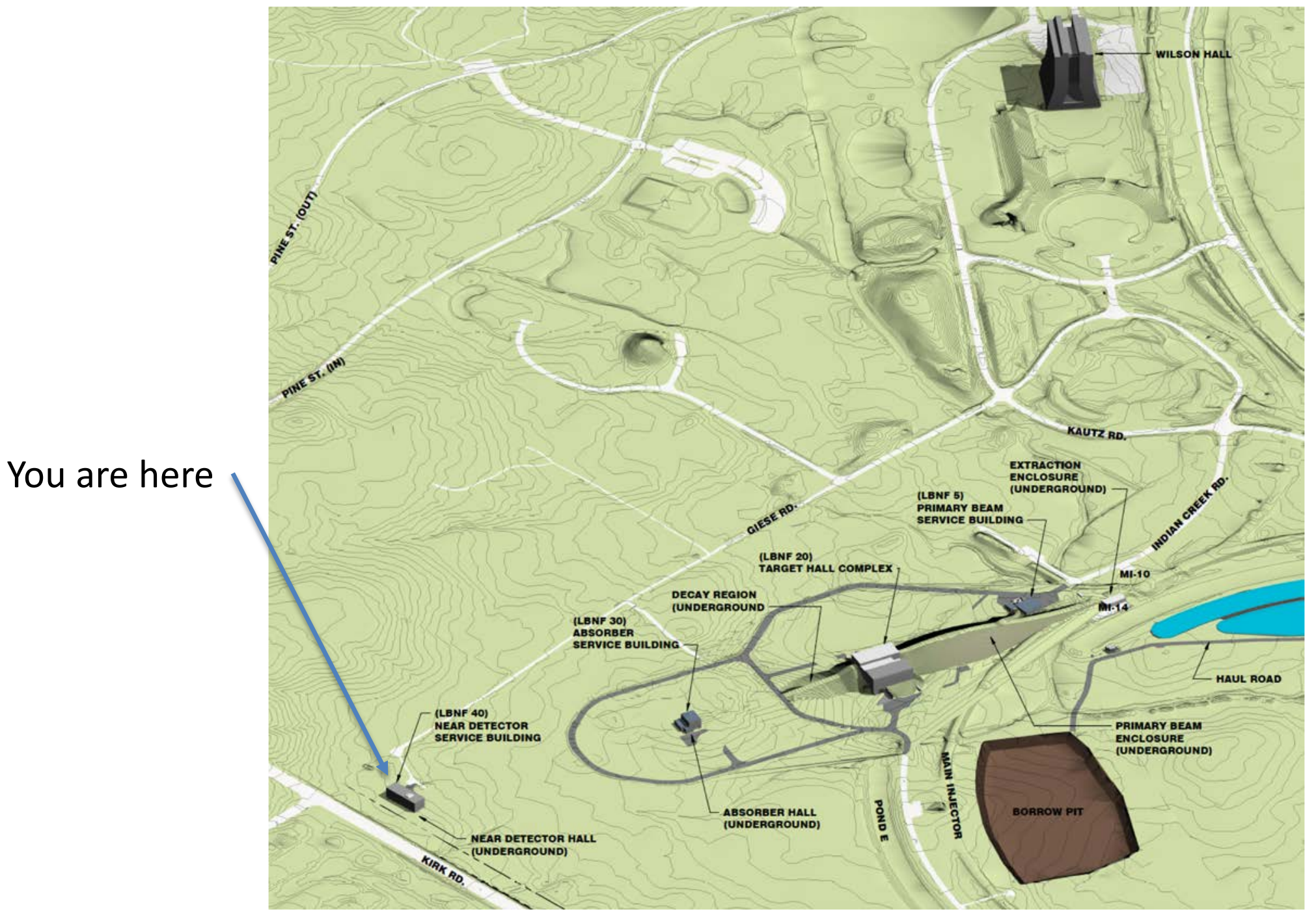




\section{Looking underground}

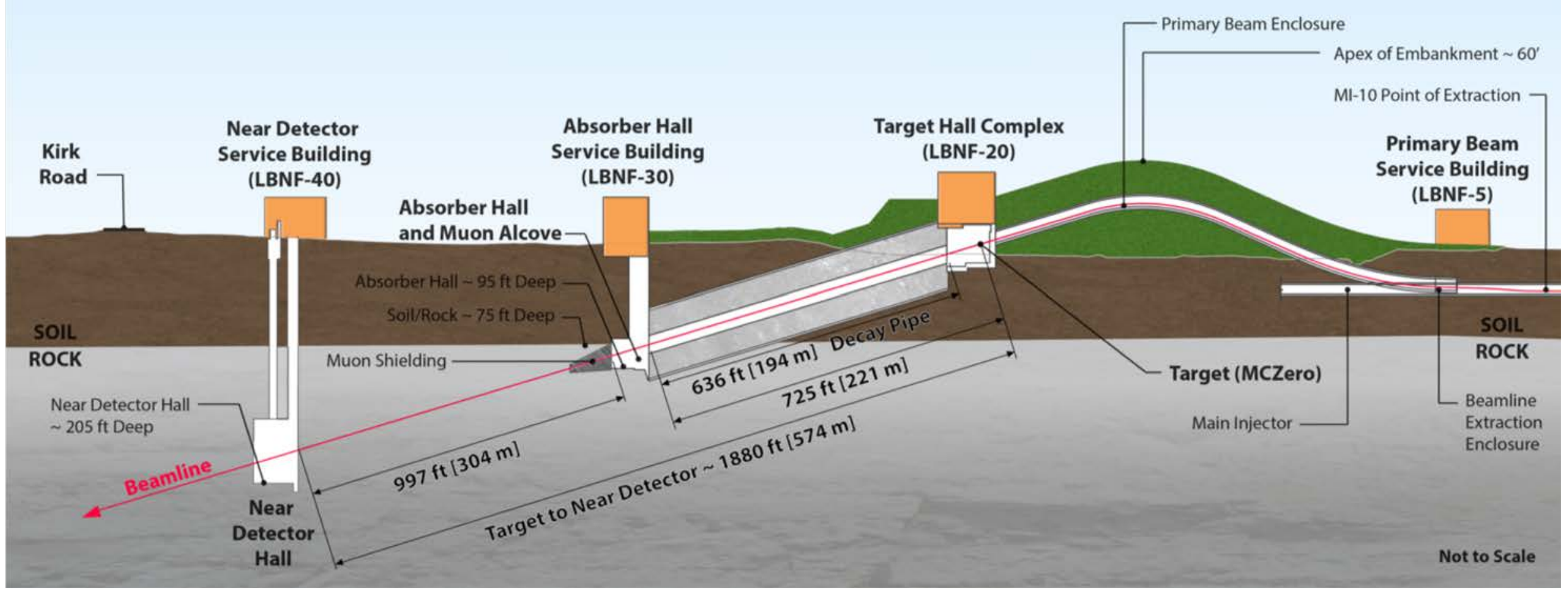




\section{Service building: Reference design}

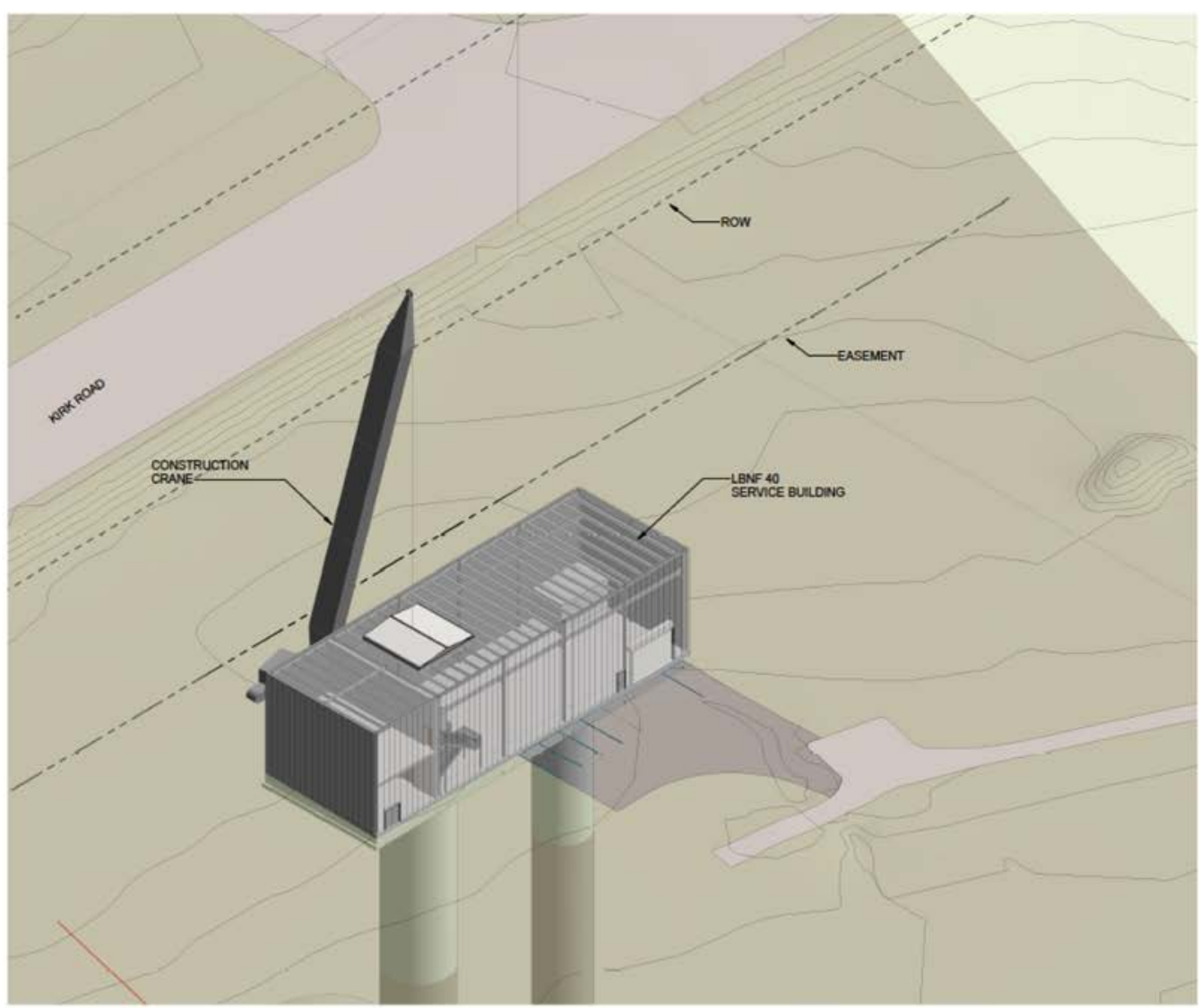

3D PRESENTATION AT GRADE LEVEL

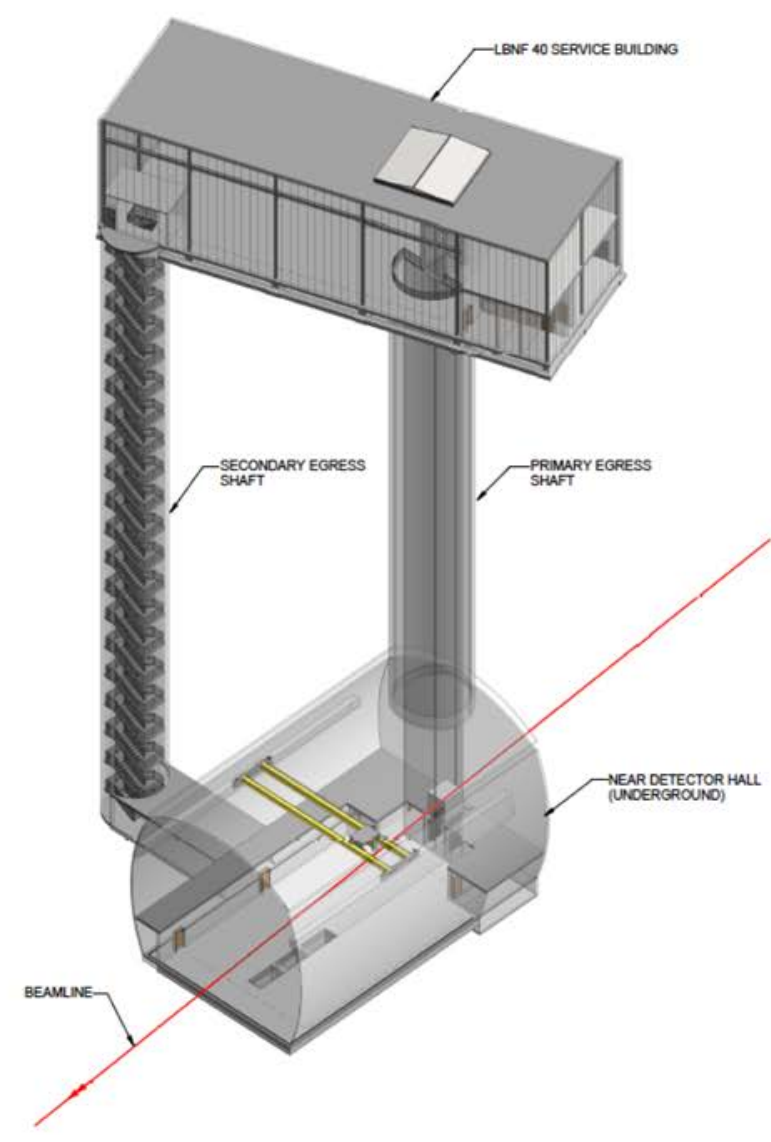

3D PRESENTATION 


\section{Flux, event rates @ ND570}

Optimized CPV tune

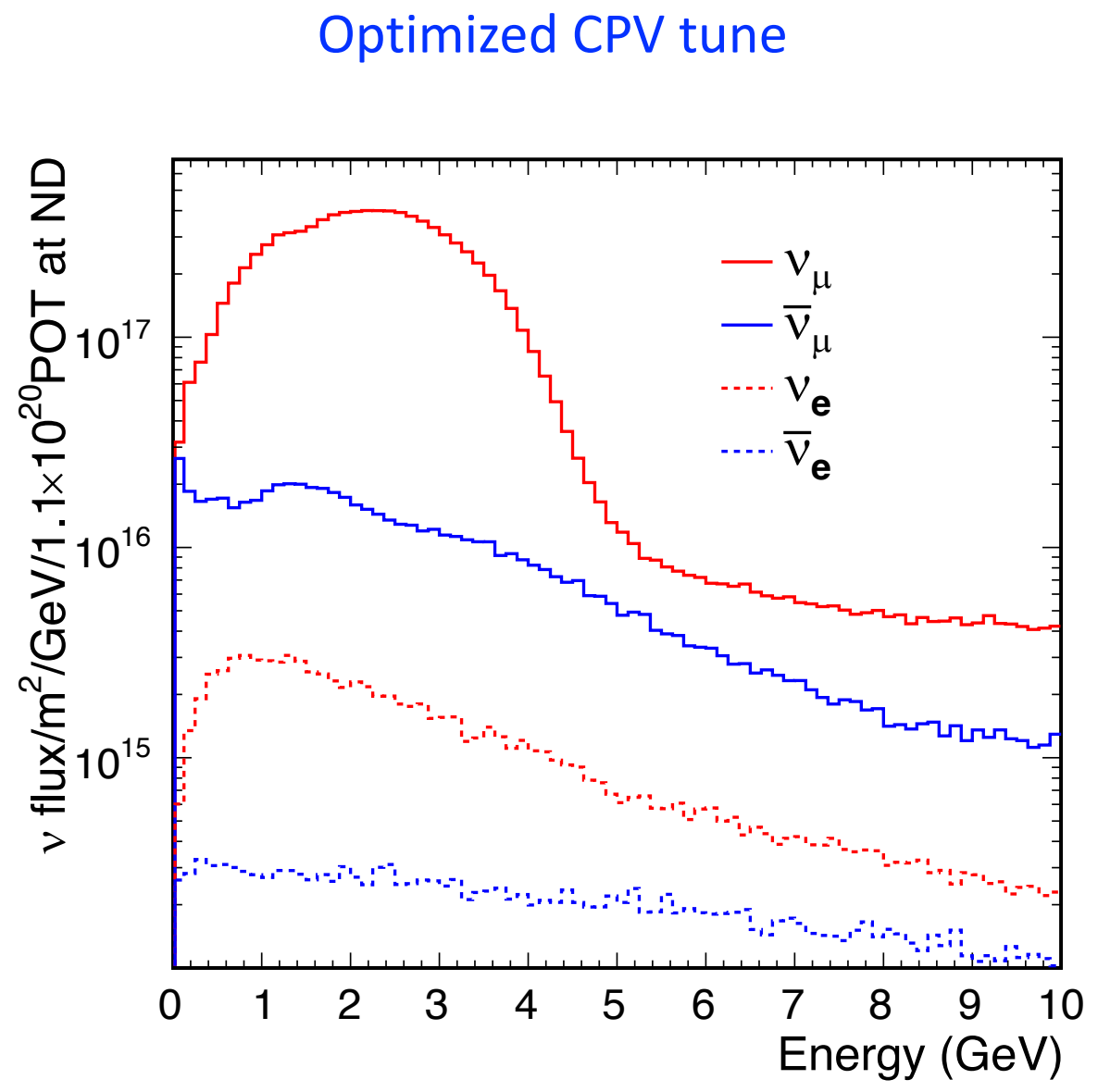

FHC, Events/ton_Ar-year

\begin{tabular}{l|c}
\hline Event class & Number of events per ton-year \\
\hline \hline$v_{\mu}$ CC Total & $1.64 \times 10^{6}$ \\
$v_{\mu}$ NC Total & $5.17 \times 10^{5}$ \\
$v_{\mu}$ CC Coherent & $8.35 \times 10^{3}$ \\
$v_{\mu}$ NC Coherent & $4.8 \times 10^{3}$ \\
$v_{\mu}$ - electron elastic & 135 \\
$v_{\mu}$ CC $\pi^{0}$ inclusive & $4.47 \times 10^{5}$ \\
$v_{\mu}$ NC $\pi^{0}$ inclusive & $1.96 \times 10^{5}$ \\
$v_{\mu}$ Low v $(250 \mathrm{MeV})$ & $2.16 \times 10^{5}$ \\
$v_{\mu}$ Low v $(100 \mathrm{MeV})$ & $7.93 \times 10^{4}$ \\
$\bar{v}_{\mu}$ CC Coherent $(\bar{v}$ mode $)$ & $6.90 \times 10^{3}$ \\
\hline$v_{e}$ CC Total & $1.89 \times 10^{4}$ \\
$v_{e}$ NC Total & $5.98 \times 10^{3}$ \\
$v_{e}$ CC Coherent & 93 \\
$v_{e}$ NC Coherent & 52 \\
\hline \hline
\end{tabular}




\section{Beam systematics}

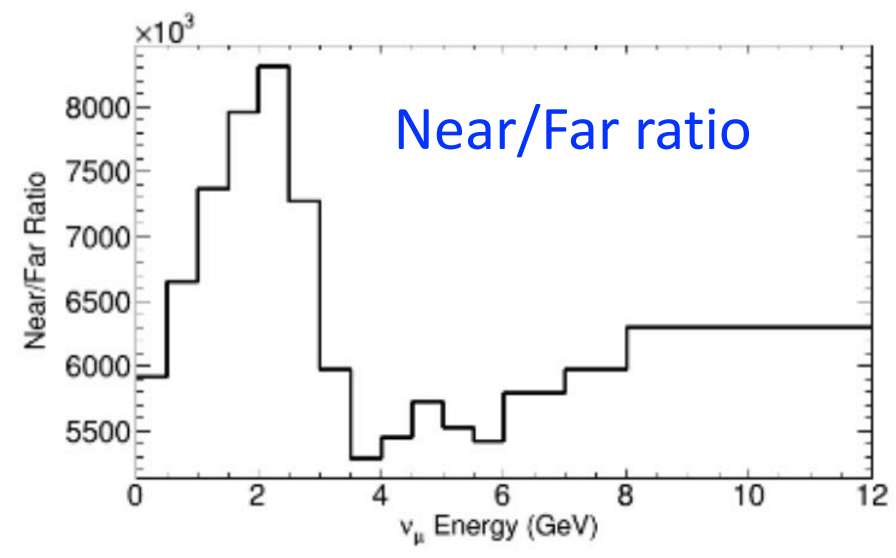

Flux uncertainty at ND

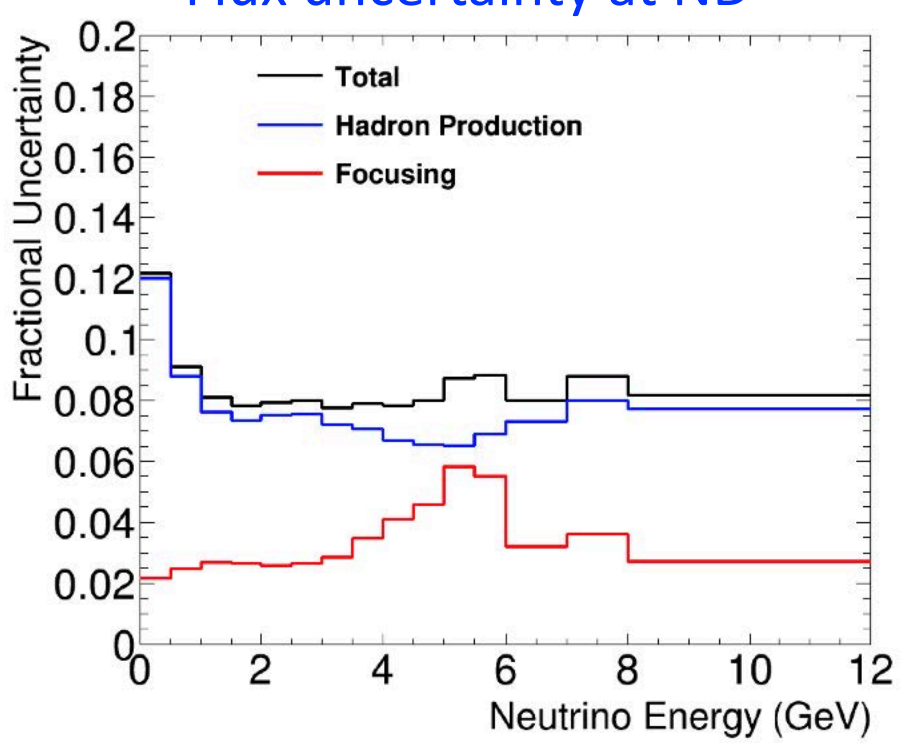

Near/Far ratio uncertainty

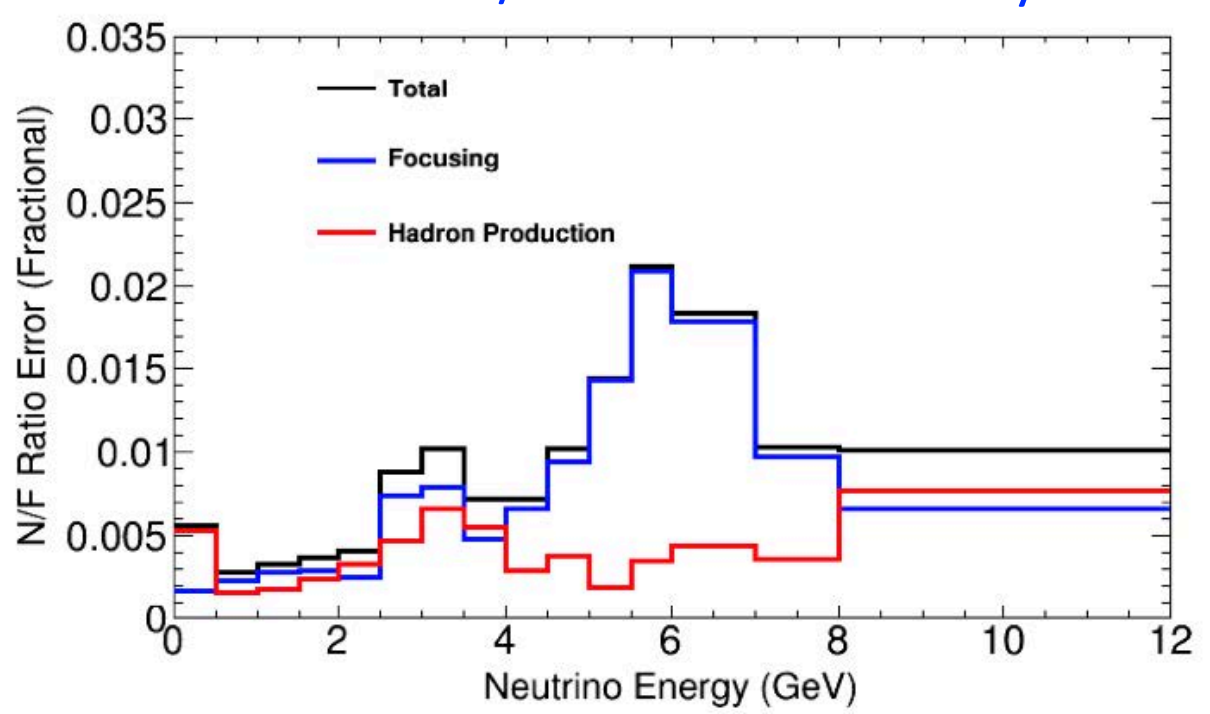




\section{Beam systematics II: Instrumentation}

- Work continues on understanding beam

- Hadron production measurements

- Flux spectrometer

- Exact mock up of LBNF target horn system with multiparticle spectrometer, PID, etc.

- EMPAHTIC

- Uses the FNAL Test Beam Facility (FTBF), either MTest or Mcenter

- Beam line instrumentation

- Muon monitors

- Conventional

- Diamond

- Muon total absorption

- Transition radiation detector

- RF-based hadron monitor 


\section{Measuring the \# of events, near \& far}

- Oscillation probabilities

$$
P_{v_{\mu} \rightarrow v_{e}}\left(E_{v}\right)=\frac{\phi_{v_{e}}^{\text {far }}\left(E_{v}\right)}{\phi_{v_{\mu}}^{\text {far,no-osc }}\left(E_{v}\right)}=\frac{\phi_{v_{e}}^{\text {far }}\left(E_{v}\right)}{\phi_{v_{\mu}}^{\text {near }}\left(E_{v}\right) * F_{\text {far } / \text { near }}\left(E_{v}\right)}
$$

- Number of events/energy spectrum

$$
\frac{d N_{v}^{d e t}}{d E_{v}}=\phi_{v_{\mu}}^{\operatorname{det}}\left(E_{v}\right) * \sigma_{v_{\mu}}^{A r}\left(E_{v}\right)
$$

- In reality

$$
\frac{d N_{v}^{d e t}}{d E_{r e c}}=\int \phi_{v}^{d e t}\left(E_{v}\right) * \sigma_{v}^{\text {target }}\left(E_{v}\right) * T_{v_{\mu}}^{d e t}\left(E_{v}, E_{r e c}\right) d E_{v}
$$

- Folding of detector effects

- Prevents (easy) cancellations of many systematic effects

- Needs unfolding 


\section{Details}

- Oscillation signal

$$
\left.\frac{d N_{v_{e}}^{f a r}}{d E_{v}}\right|_{\frac{d N_{v_{\mu}}^{\text {near }}}{d E_{v}}}=P_{v_{\mu} \rightarrow v_{e}}\left(E_{v}\right) * \frac{\sigma_{v_{e}}^{A r}\left(E_{v}\right)}{\sigma_{v_{\mu}}^{A r}\left(E_{v}\right)} * F_{f a r / \text { near }}\left(E_{v}\right)
$$

- Near muon/electron ratio

$$
\frac{d N_{v_{e}}^{\text {near }}}{d E_{v}} / \frac{d N_{v_{\mu}}^{\text {near }}}{d E_{v}}=\left.\frac{\sigma_{v_{e}}^{A r}\left(E_{v}\right)}{\sigma_{v_{\mu}}^{A r}\left(E_{v}\right)} * \frac{\phi_{v_{e}}^{\text {near }}\left(E_{v}\right)}{\phi_{v_{\mu}}^{\text {near }}\left(E_{v}\right)}\right|_{\text {Uncertainty }}
$$

- Need to know

- Flux \& cross section ratios

- Far/near extrapolation 


\section{Details II}

- Since $E_{v}$ rec is not equal to $E_{v}$

- Need to understand

- Detector effects in near and far detector

- Relation of visible to neutrino energy

- NEUTRONS

- Cross section ratios

- Near to far flux extrapolation

- Flux normalisation provides some cancellation

- Shape is important, however 


\section{Near Detector needs to measure:}

- ND Fluxes

$$
\phi_{v_{x}}^{\text {near }}\left(E_{v}\right)
$$

- Prior constrained $5-10 \%$

- Total and differential cross sections on Argon

$$
\frac{d^{n} \sigma_{v_{x}}^{A r}}{d a d b d c \ldots}\left(E_{v}\right) \quad \text { (Largely unknown) }
$$

- True to reconstruction "matrix"

$$
T_{v_{x}}^{\text {far }}\left(E_{v}, E_{r e c}\right) \text { and } T_{v_{x}}^{\text {near }}\left(E_{v}, E_{r e c}\right)
$$

- Depends on: Detector effects, xsections, nuclear effects

- Approach

- Measure as many exclusive differential cross sections with as much precision as possible

$$
\frac{d N}{d X_{\text {rec }}}=\int \phi_{v_{\mu}}^{\text {near }}\left(E_{v}\right) \frac{d \sigma_{v_{\mu}}^{A r}}{d X}\left(E_{v}\right) T_{v_{\mu}}^{\text {near }}\left(E_{v}, X, X_{r e c}\right) d E_{v} d X
$$




\section{Flux measurements}

- Primary thrust within DUNE near detector suite is to do measurements on $\operatorname{Ar}$ (Liquid and gas)

- Proposed measurements

- Neutrino-electron scattering (LAr)

- Low-v method (liquid and gas)

- Coherent Scattering(liquid and gas)

- $v_{l}+N \rightarrow l^{-}+N+\pi^{+}$

- $\bar{v}_{l}+N \rightarrow l^{+}+N+\pi^{-}$

- Measurements on hydrogen ( $\mathrm{CH}$ and gas)

- $v_{l}+p \rightarrow l^{-}+\Delta^{++} \rightarrow l^{-}+p+\pi^{+}$

- $\bar{v}_{l}+p \rightarrow l^{+}+\Delta^{0} \rightarrow l^{+}+p+\pi^{-}$ 


\section{DUNE Near Detector Concept Study}

- The Near Detector Concept Study explored the requirements, technology and physics performance of a number of options for the near detector.

- It was an approximately 2 year effort which included workshops at Fermilab and CERN and targeted study by 6 working groups

- The final report, providing recommendations for the near detector complex, was submitted to the DUNE Executive Board in July 2018.

- At the end of August, the Executive Board approved the recommendations that were proposed. 


\section{Main Near Detector Recommendations}

- The recommended concept is a near detector suite consisting of a LArTPC (not in a magnetic field), a HPgTPC in a magnet, and a 3DST.

- The design of a mobile LAr detector that can make measurements at one or more off-axis positions should go forward (DUNE-PRISM). Study option of moving HPgTPC also

- The experimental floor area should be at least $42.5 \mathrm{~m} \times 17 \mathrm{~m}$ and the hook height must be at least $13 \mathrm{~m}$, measured from the floor. The minimum lateral dimension of hall needs further study, and will ultimately be settled in EFIG.

- The option of filling the HPgTPC with hydrogen should also be investigated. 


\section{Multi-pronged approach}

- Prong I: State-of-the-art Ar detectors:

- LAr ( 75t fiducial target mass), non-magnetized

- Pixelated (raw 3D data)

- Optically segmented

- Neutron tagging

- Multi-purpose Detector (MPD)

- High-Pressure (10ATM) gas TPC (HPgTPC) (1t fiducial target mass)

- In $\sim 0.5 \mathrm{~T}$ field (magnetic spectrometer)

- Surrounded by high-performance ECAL and muon tagger

- Prong II: DUNE-PRISM

- Move LAr and possible MPD off axis

- Prong III: 3 dimensional scintillator (CH) tracker (3DST) (4t)

- Interactions on protons and carbon

- Magnetized

- With external tracking and ECAL 


\section{LAr: ArgonCube}

- Underlying principles

- True Raw 3D readout - in a sense, the first true LArTPC

- Pad readout, no wires

- $\mathrm{S} / \mathrm{N}>>$ than in conventional LAr TPCs

- Better energy resolution and better pointing resolution

- Modular, highly segmented

- Short drift $\Rightarrow$ little diffusion, low high voltage, less sensitive to impurities

- Optically isolated modules $\Rightarrow$ more effective use of scintillation light 


\section{LAr: ArgonCube design}

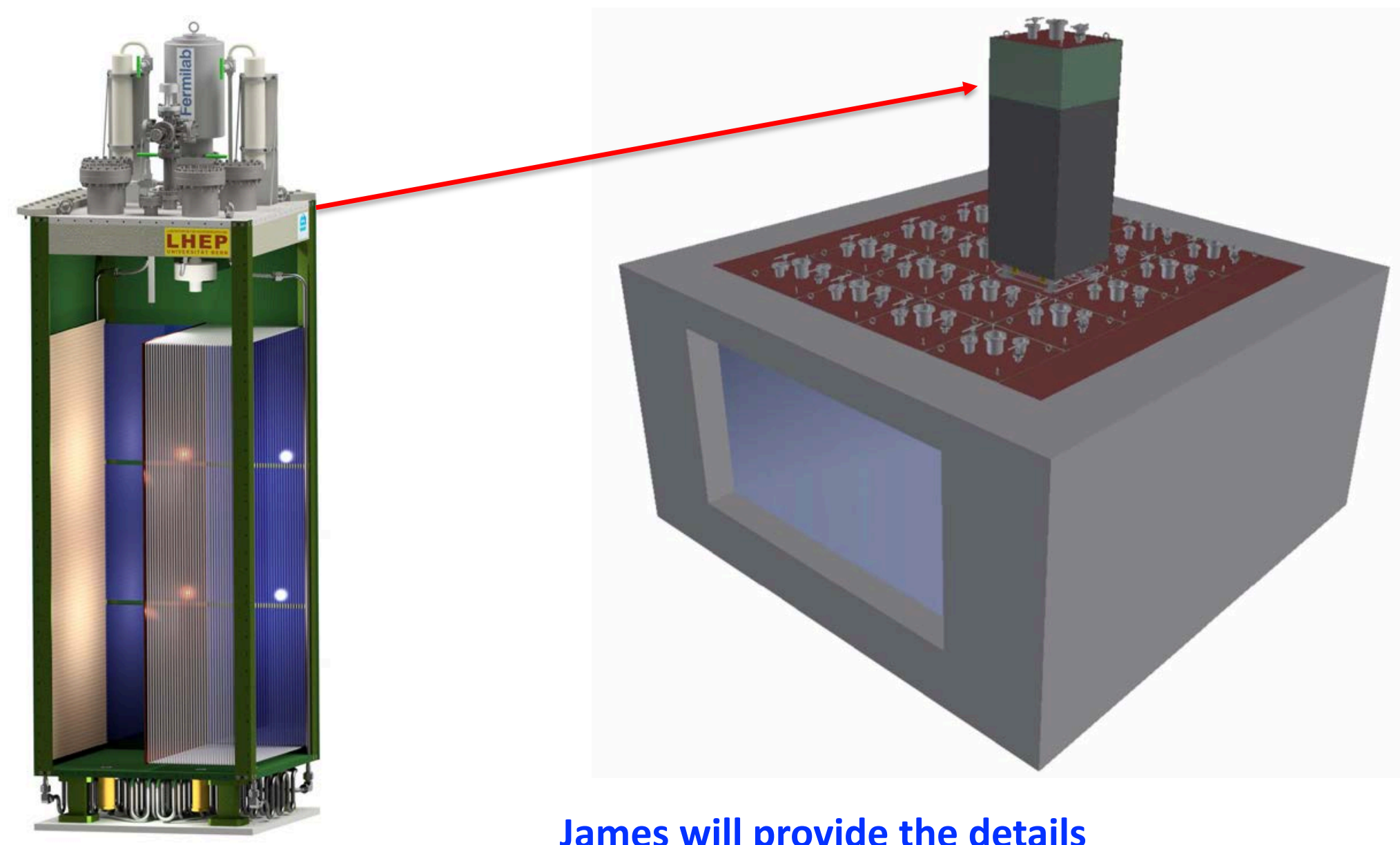

James will provide the details 


\section{Multi-purpose detector}

- Central component is a large gas TPC operating at $10 \mathrm{Atm}$ (HPgTPC)

- Copy of ALICE TPC ( $5 \mathrm{~m}$ in diameter X $5 \mathrm{~m}$ long active)

- Re-use the ALICE readout chambers were are being replaced during the current long shutdown (\& engineering)

- HPgTPC surrounded by high-performance ECAL following designs developed in the CALICE program

- 0.5T B field

- Superconducting design looks most promising

- Open geometry

- Muon tagger outside coils

- MPD is essentially a Collider Detector design 


\section{MPD}

ALICE being lowered into Hall

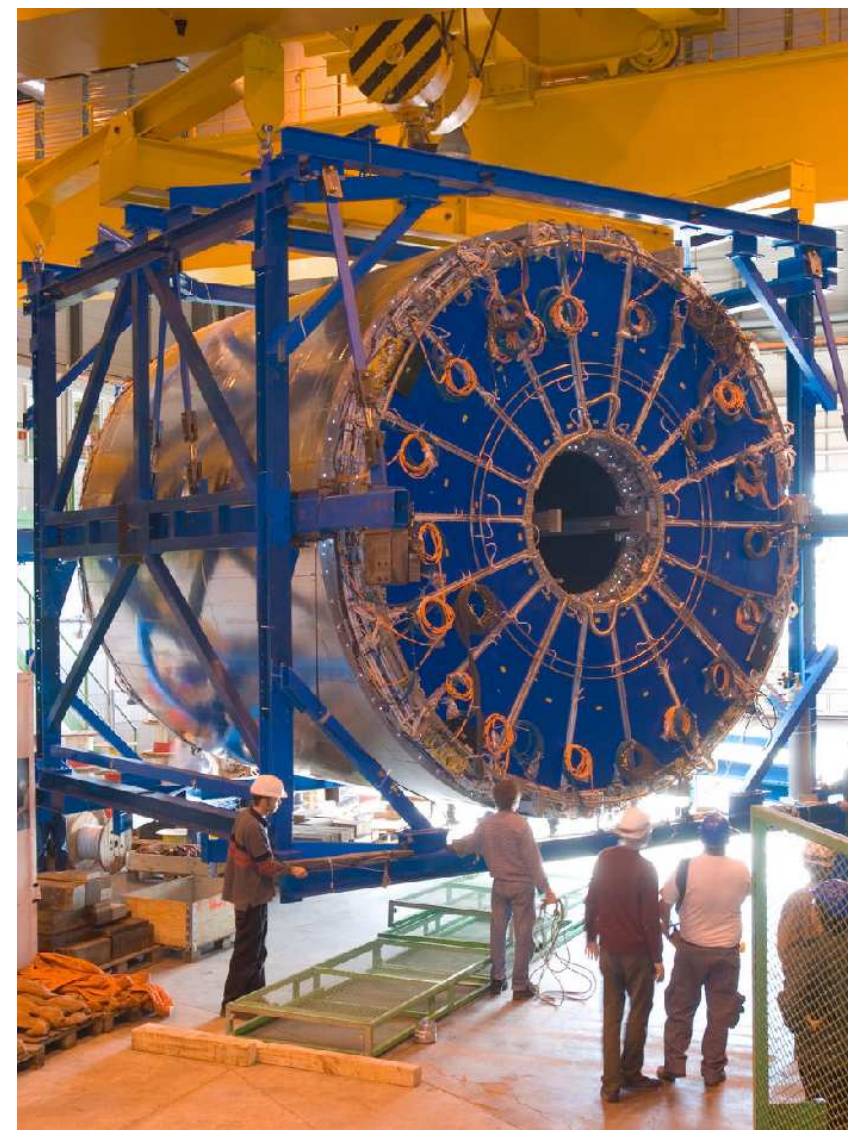

Tanaz will go into the details
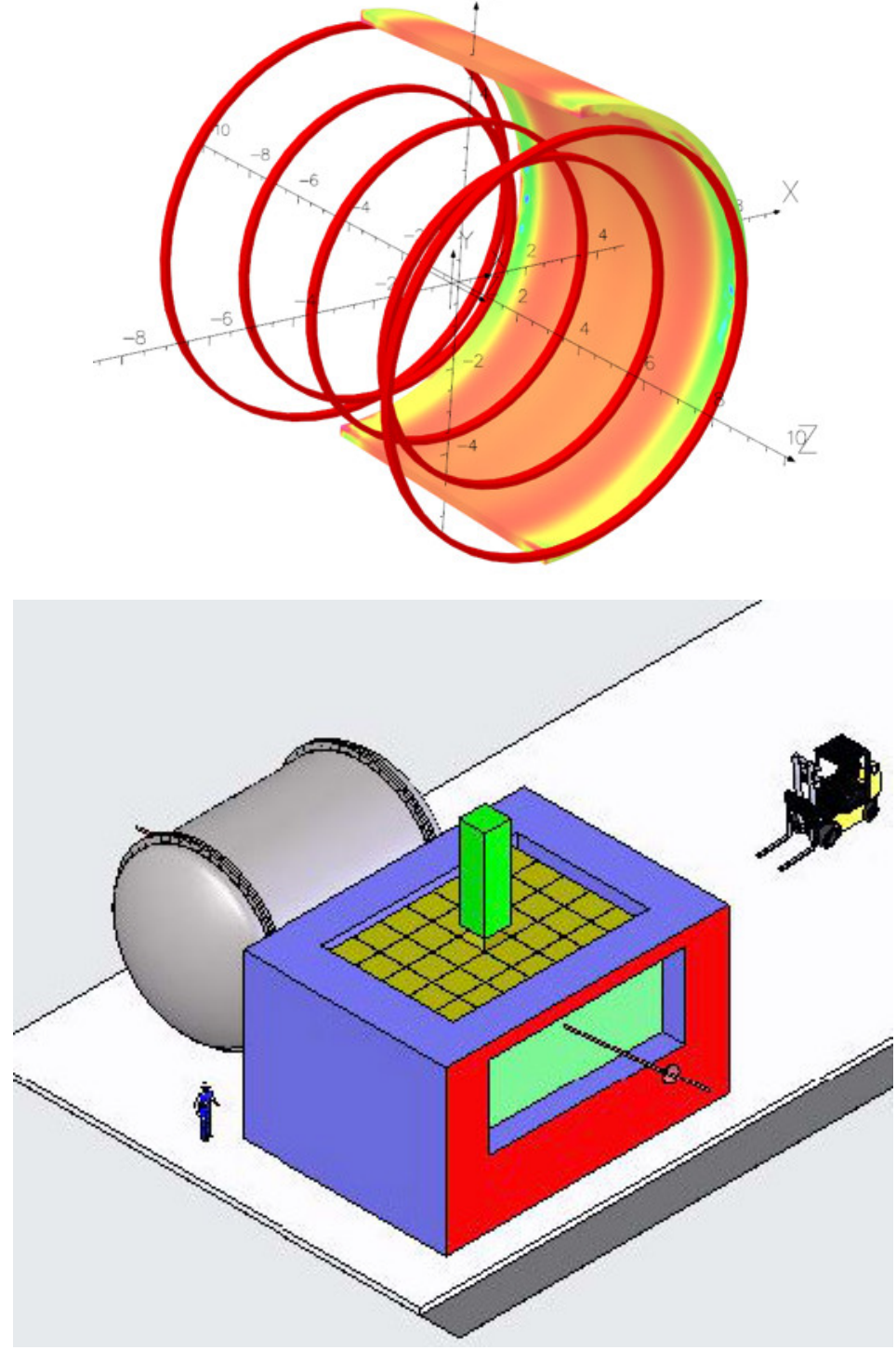
- Magnetized system complementary to MPD/HPgTPC

- Different target nucleus

- High statistics tests of neutrino models

- Connection to the existing catalog of cross section measurements on scintillator $(\mathrm{K} 2 \mathrm{~K}$, MiniBooNE, SciBooNEne, MINERvA, T2K, NOVA)

- Can remain on-axis when other detectors move off-axis

- Accurate determination of the flux

- High statistics measurement of the beam electron neutrino component

Clark will give the details

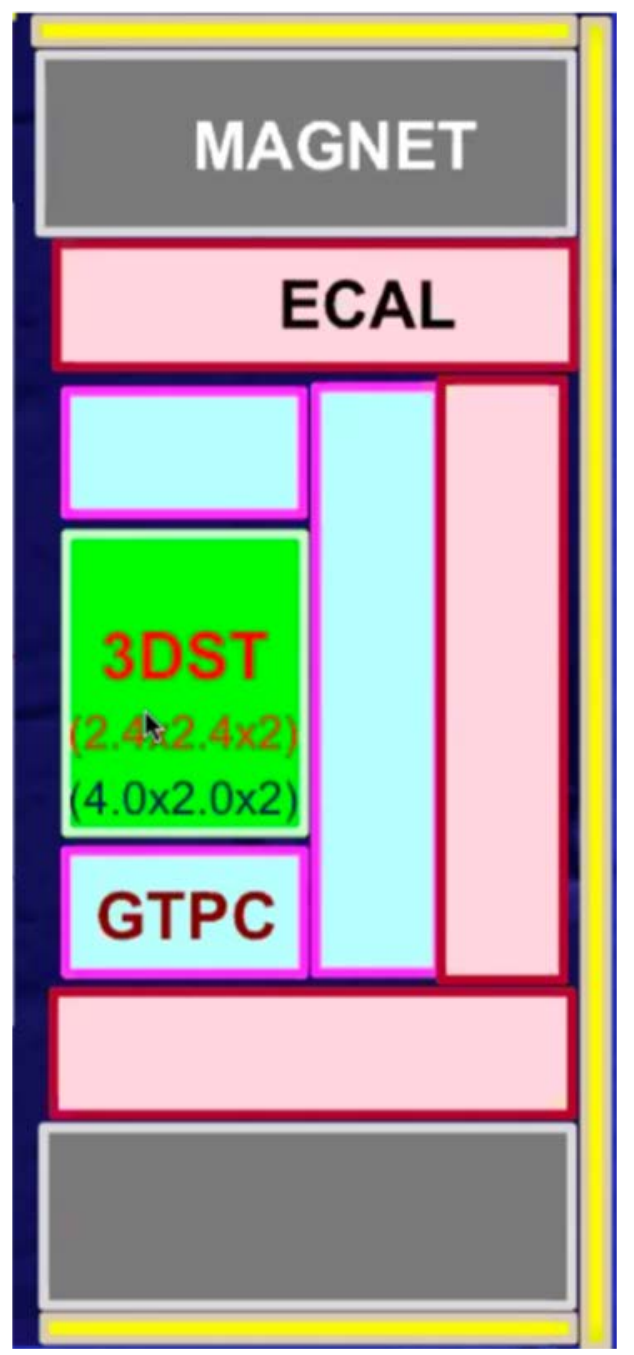




\section{DUNE-PRISM}

\section{DUNE-PRISM}

- By moving the near detector off-axis, we can measure different $E_{v}$ spectra

- The provides a new degree of freedom over which we can constrain Erec vs Etrue

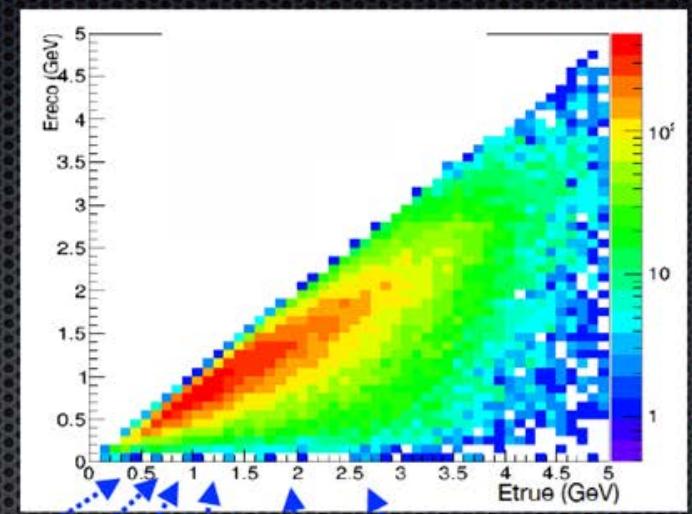

- Goal is to make measurements as similar as possible in all off-axis positions

\section{(1):}

$$
\text { : }
$$

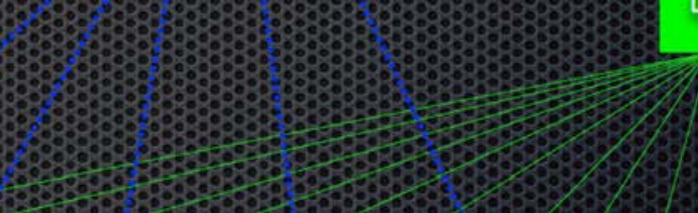

: $:$ increasing Offaxis angle

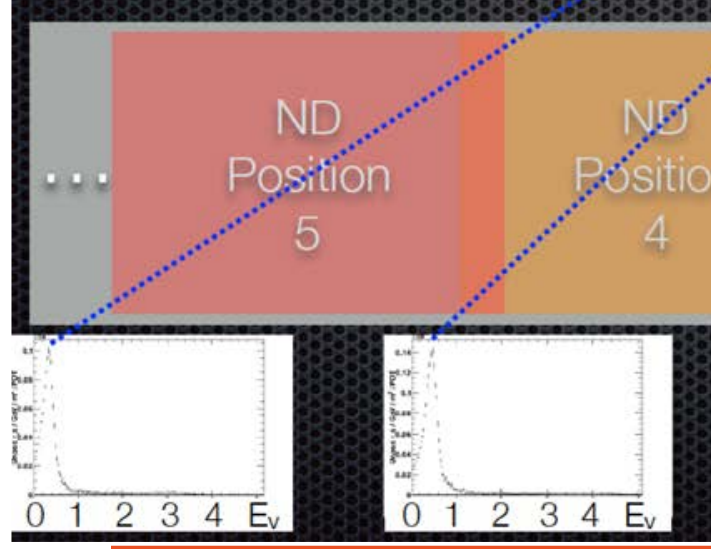

$2312 / 3 / 2018$

A. Bross I The DUNE Near Detector Complex

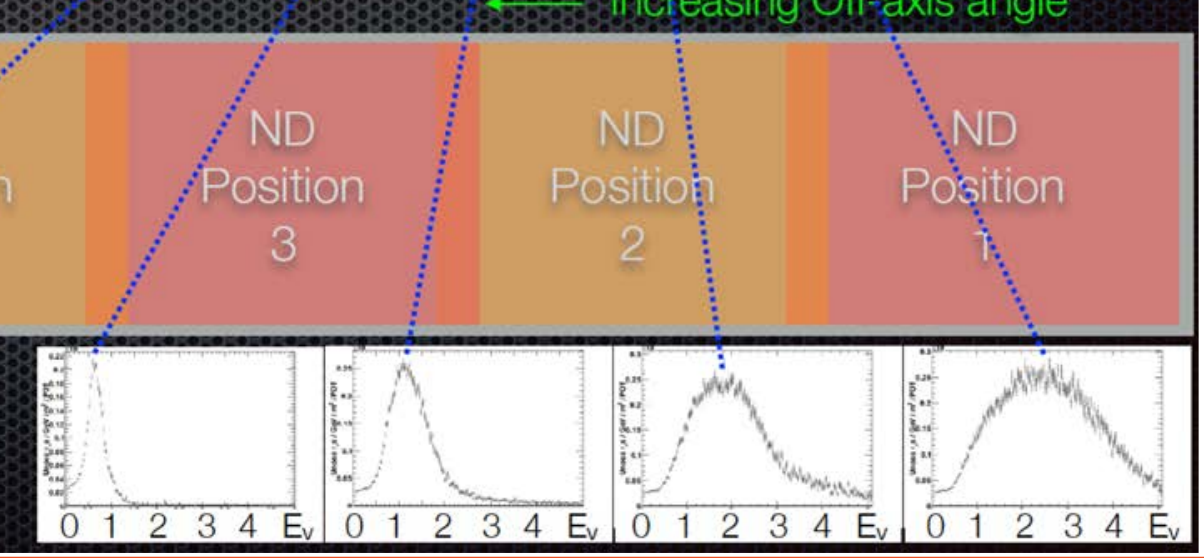

Use linear combinations to disentangle flux and $x$-section effects using different fluxes.

Cristovao will give the details

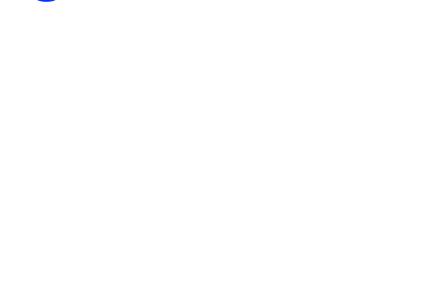




\section{Where we house all this stuff}

\section{* Fermilab}

50 Years of Discovery 


\section{Hall: Reference design (2015 CDR)}

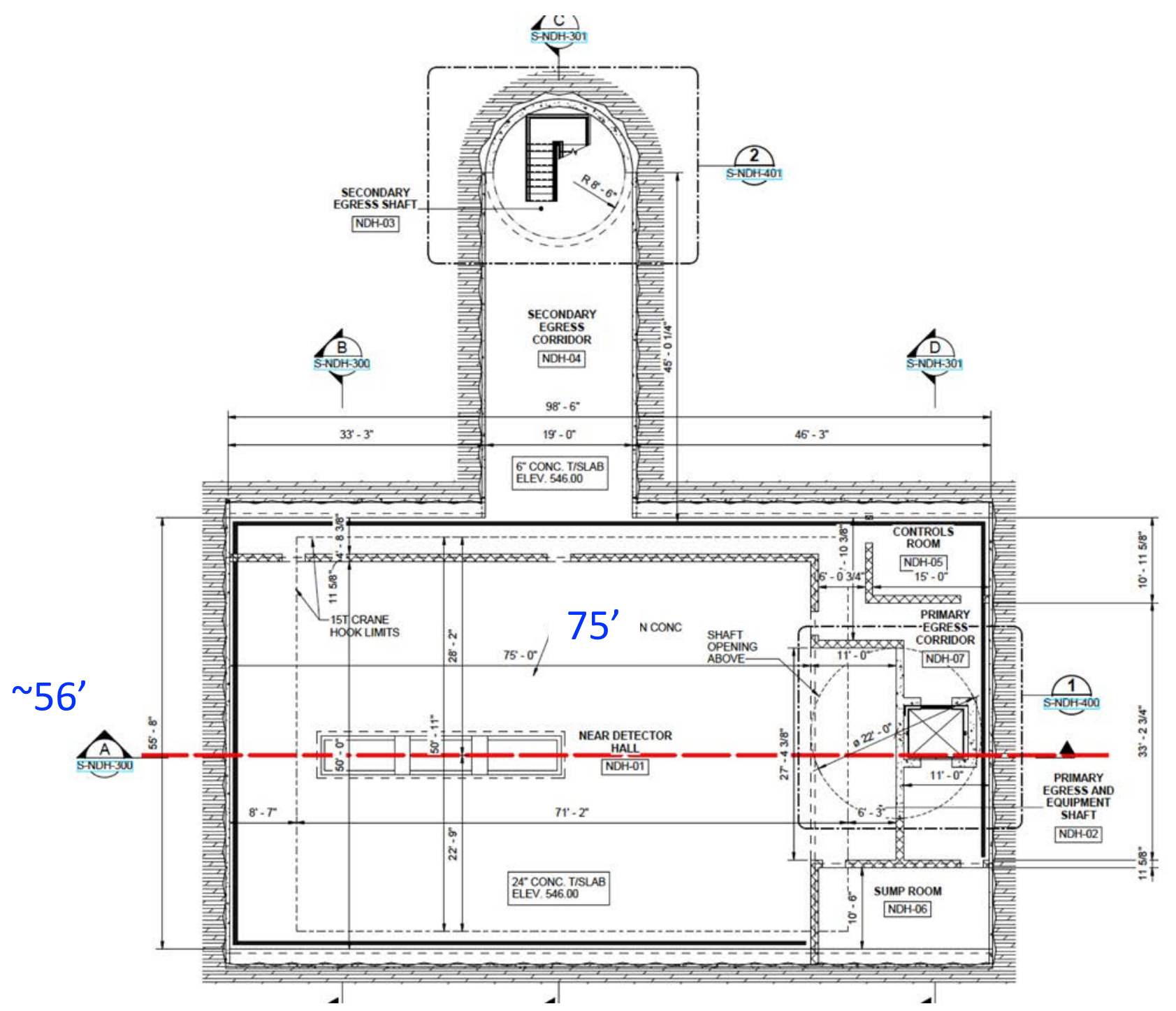




\section{Hall: Reference design II}

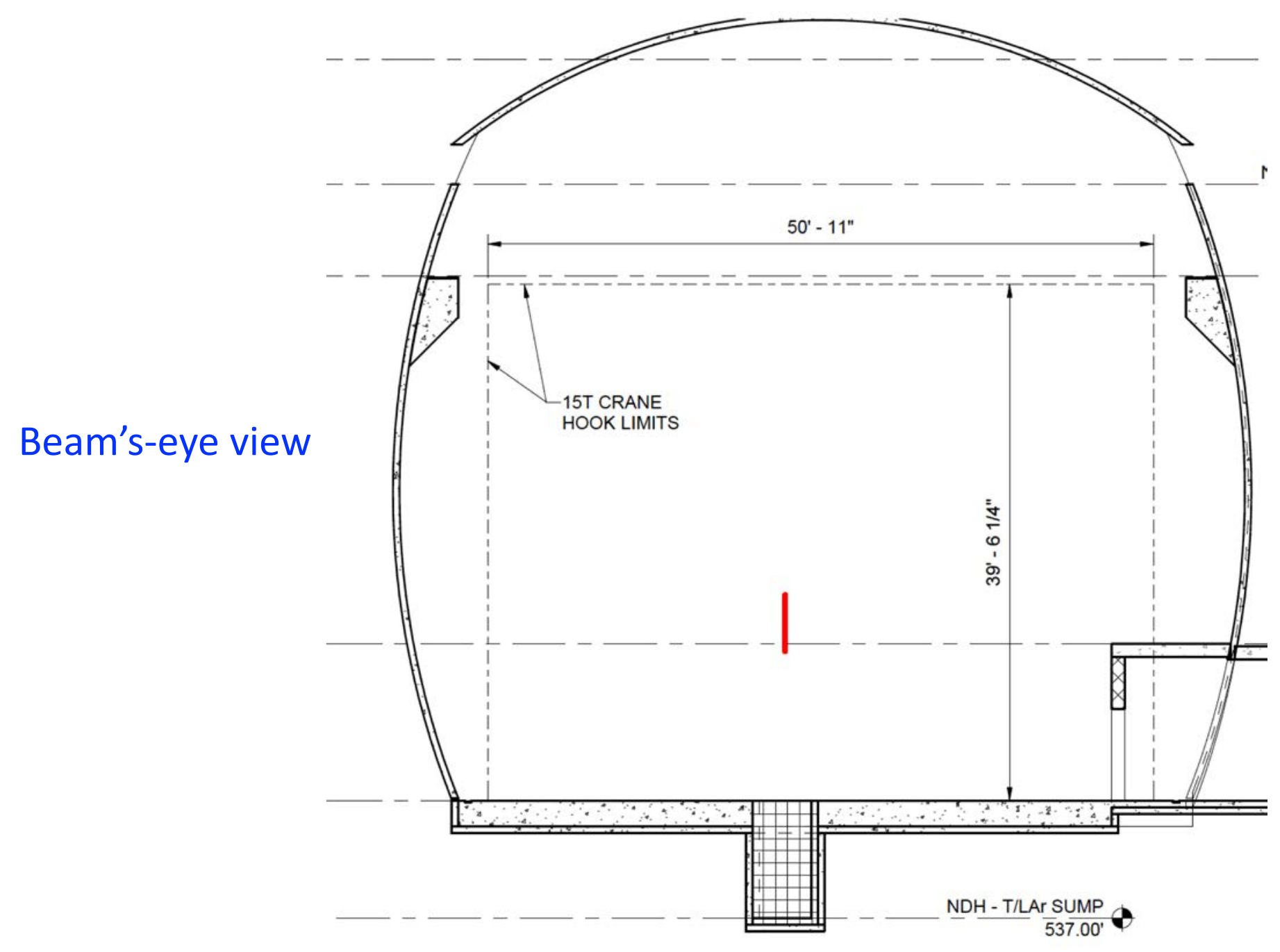




\section{Hall: Reference design III}

- It becomes obvious rather quickly that the hall reference design does not accommodate our current detector designs and run plan 


\section{Near Detector Hall: June 2018 Update}

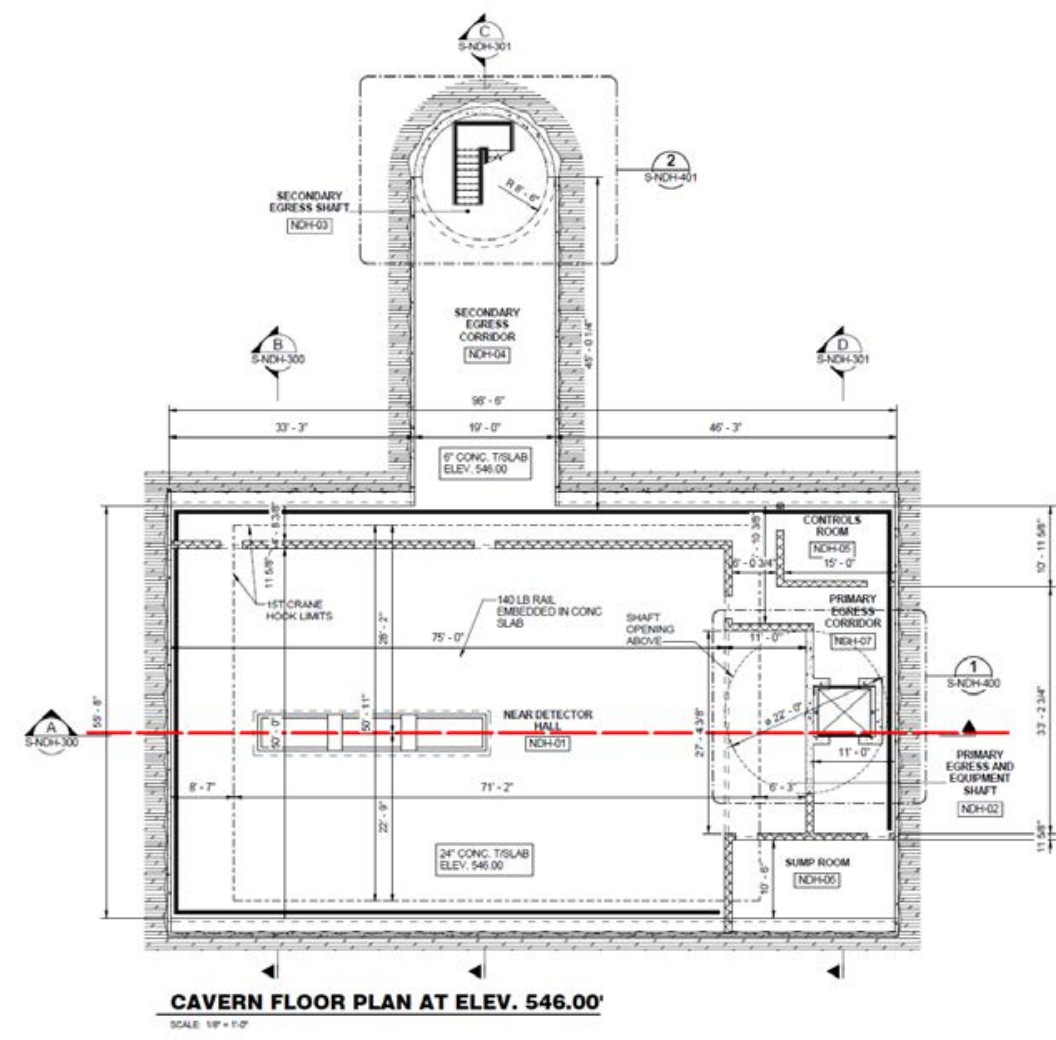

Reference ND Detector Cavern Concept: 100ft x 56ft Cavern with $75 \mathrm{ft} \times 50 \mathrm{ft}$ Detector Hall

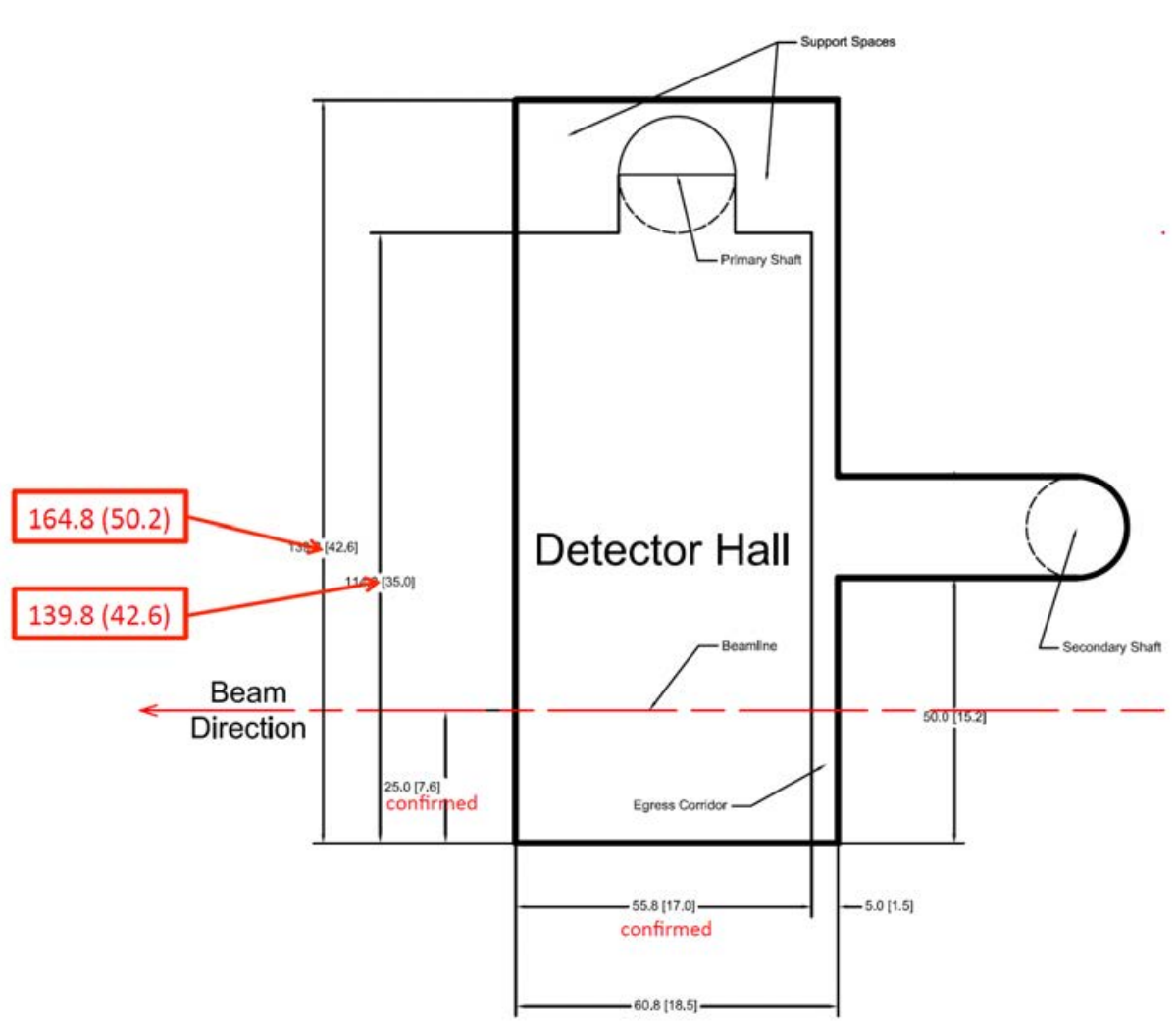

June 2018 ND Collaboration Proposal: 165ft x 61ft Cavern with $140 \mathrm{ft} \times 56 \mathrm{ft}$ Detector Hall 


\section{Primary access shaft: Reference design}

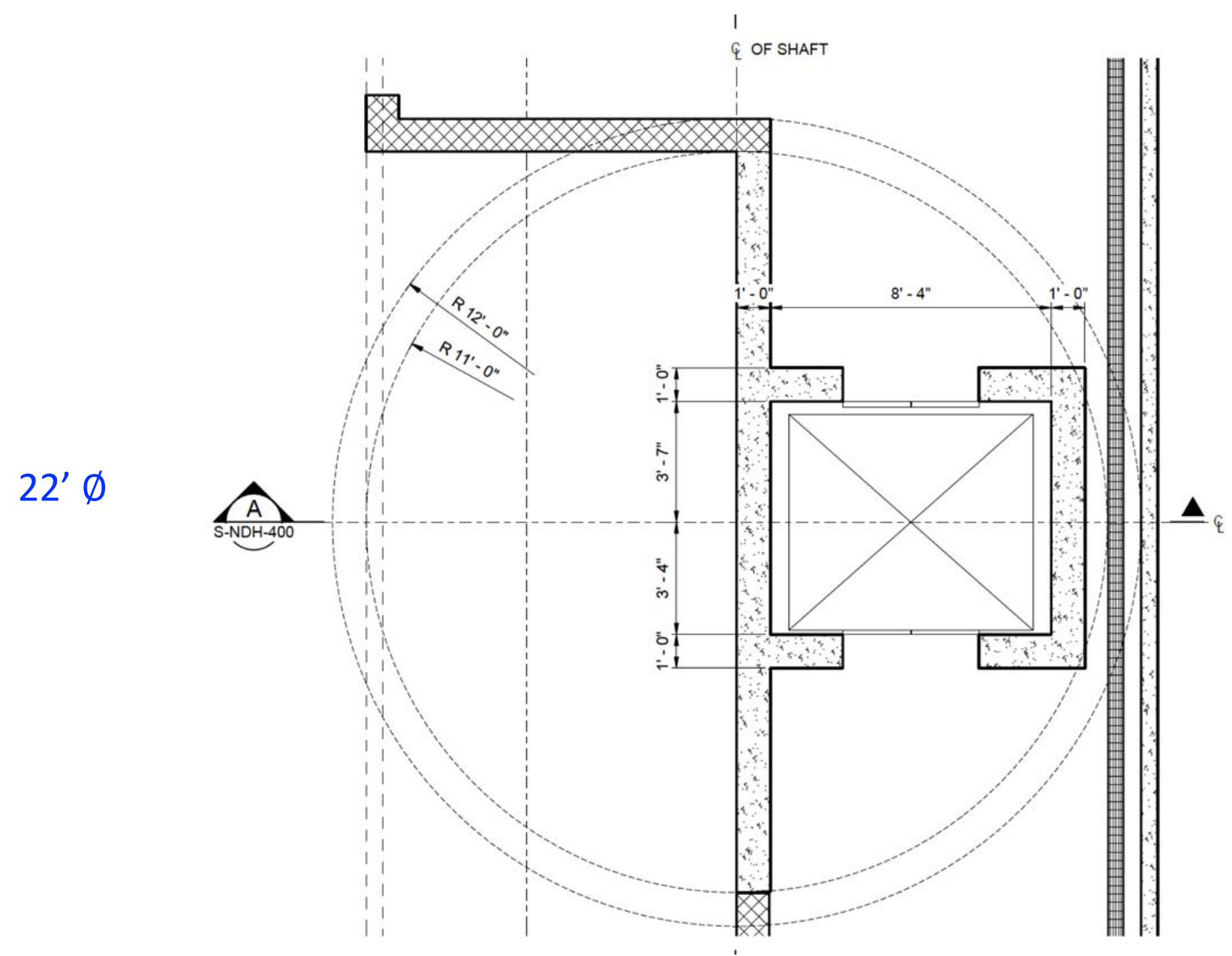




\section{Larger Shaft - Size}

- Reference shaft is 22ft ID

- Considered shaft diameters ranging from $32 \mathrm{ft}$ to $43 \mathrm{ft}$ ID

- Now looks like a 38ft ID shaft provides a minimum of $0.5 \mathrm{~m}$ clearance around HPgTPC and preserves lift/utility segment

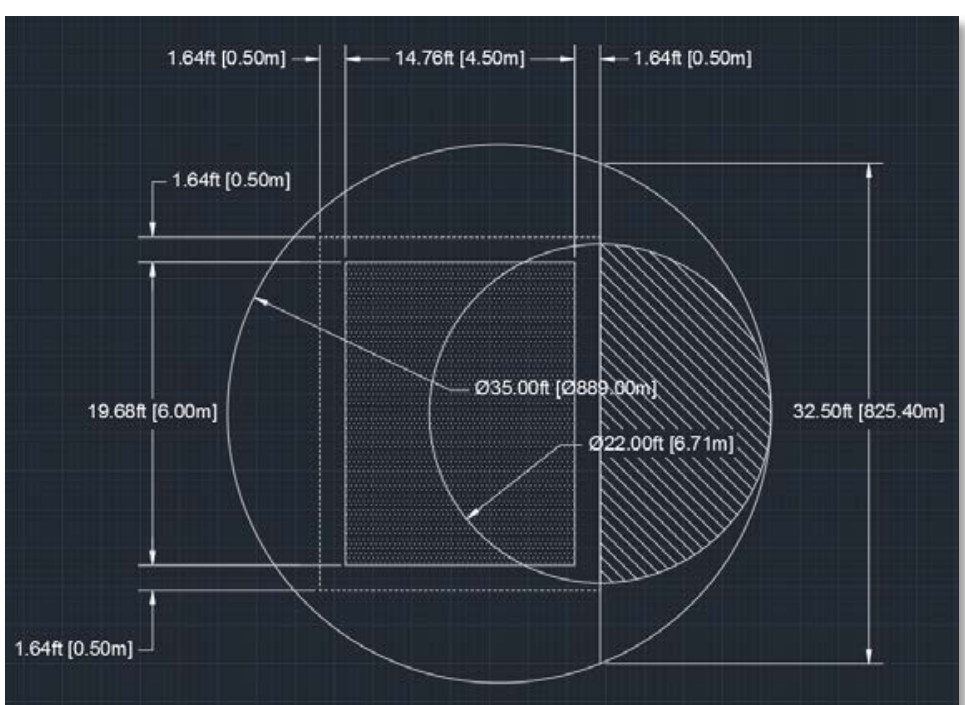

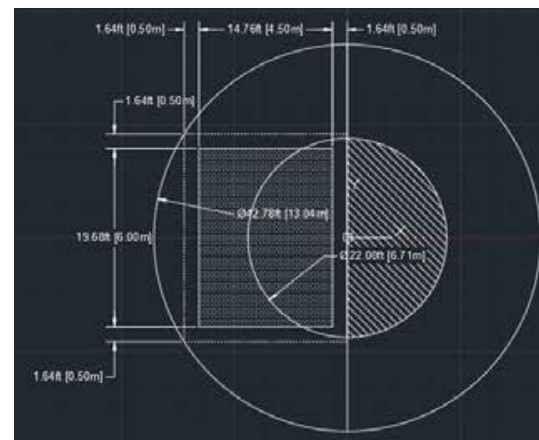

Shaft sized to keep septum wall centered in shaft - 43ft diameter

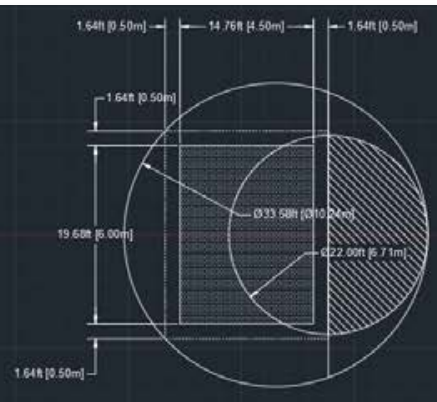

Shaft sized to preserve the cross-sectional shape of the $22 \mathrm{ft}$ diameter shaft's lift and utility segment - 34ft diameter
$35 f t$ diameter

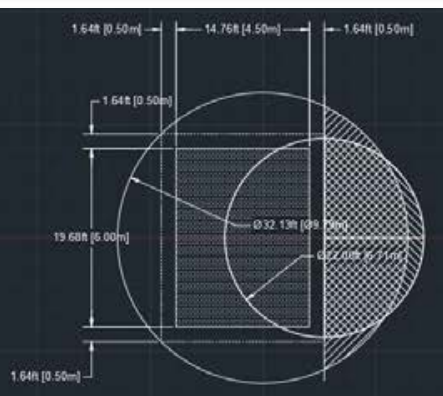

Shaft sized so that the cross-sectional area of the lift and utility segment matches that of the $22 \mathrm{ft}$ diameter shaft $32 \mathrm{ft}$ diameter 


\section{Larger cavern - cost savings?}

- Although LBNF, DUNE and Fermilab management understands the benefits of the larger cavern and access shaft for the DUNE physics program

- Trying to see if some costs can be saved while keeping the larger hall footprint and larger access shaft

- Bring Down the Roof

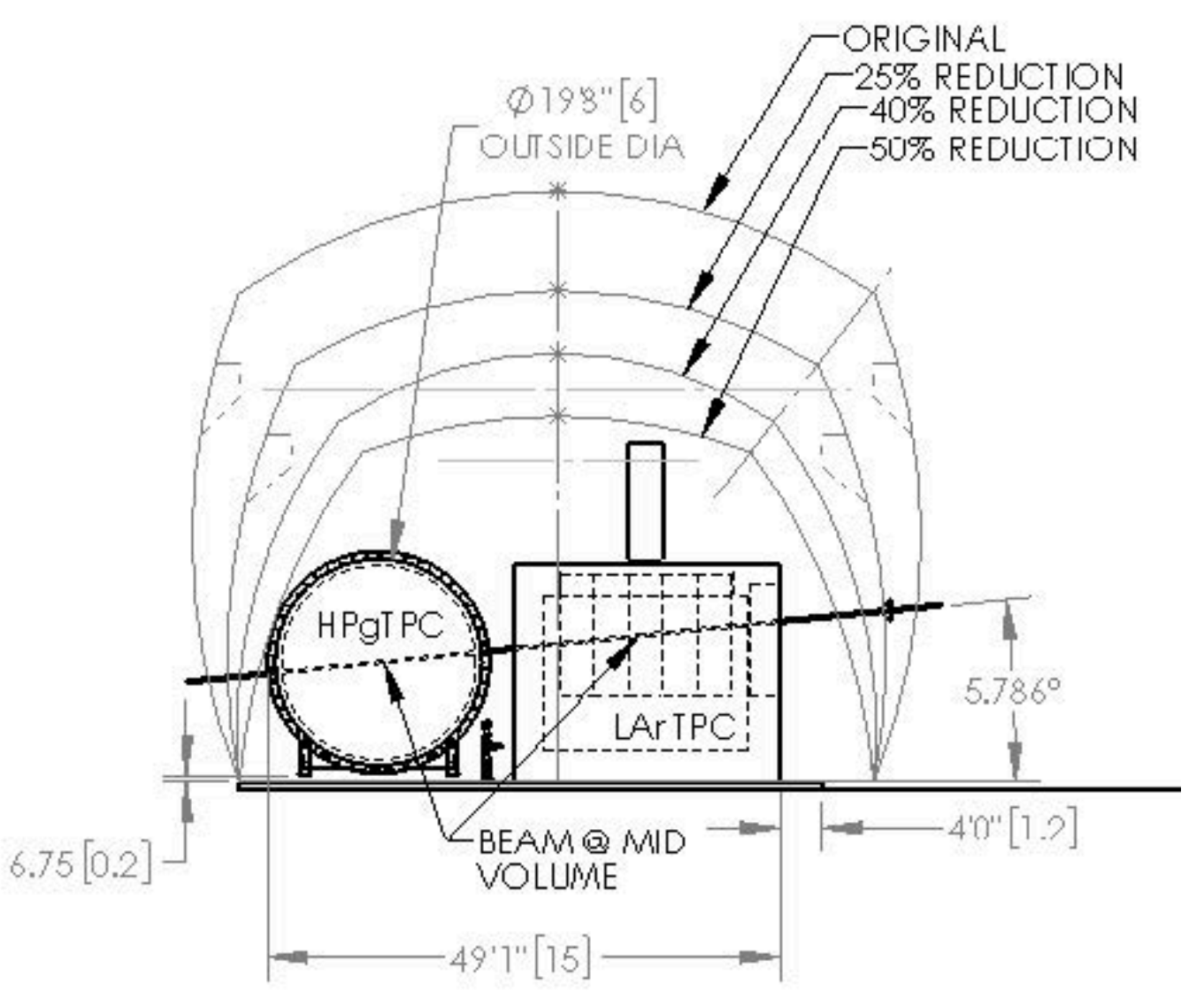




\section{One More Thing}

\section{Fermilab \\ 50 Years of Discovery}




\section{Unique capabilities of LBNF beam}

\section{High energy tune}

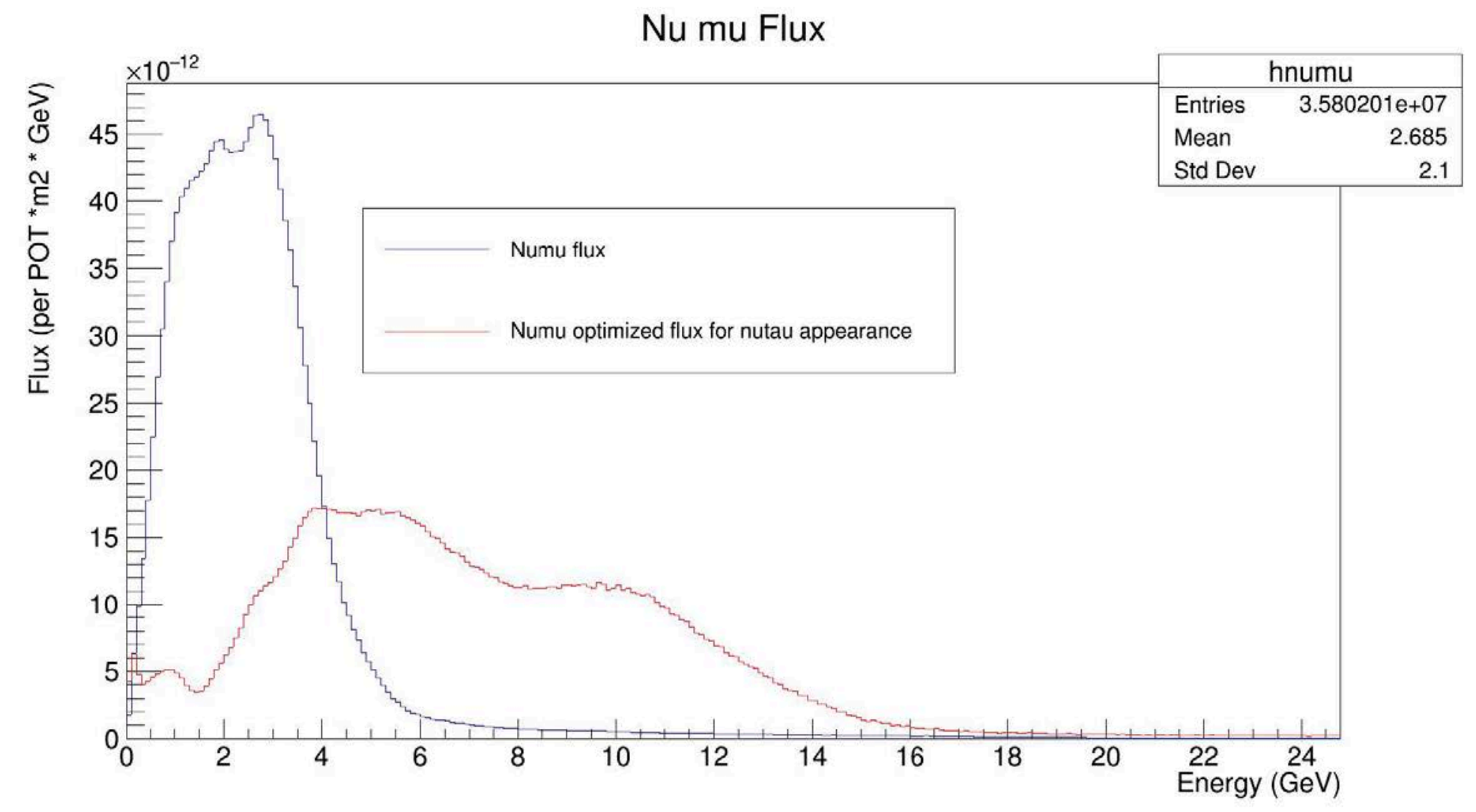




\section{$v_{\tau}$ Appearance}

- No other planned experiment/facility can study tau neutrino appearance in a neutrino beam

- What physics topics can be studied with this beam at the near site?

10X increase in $v_{\tau}$ evts in Far detector

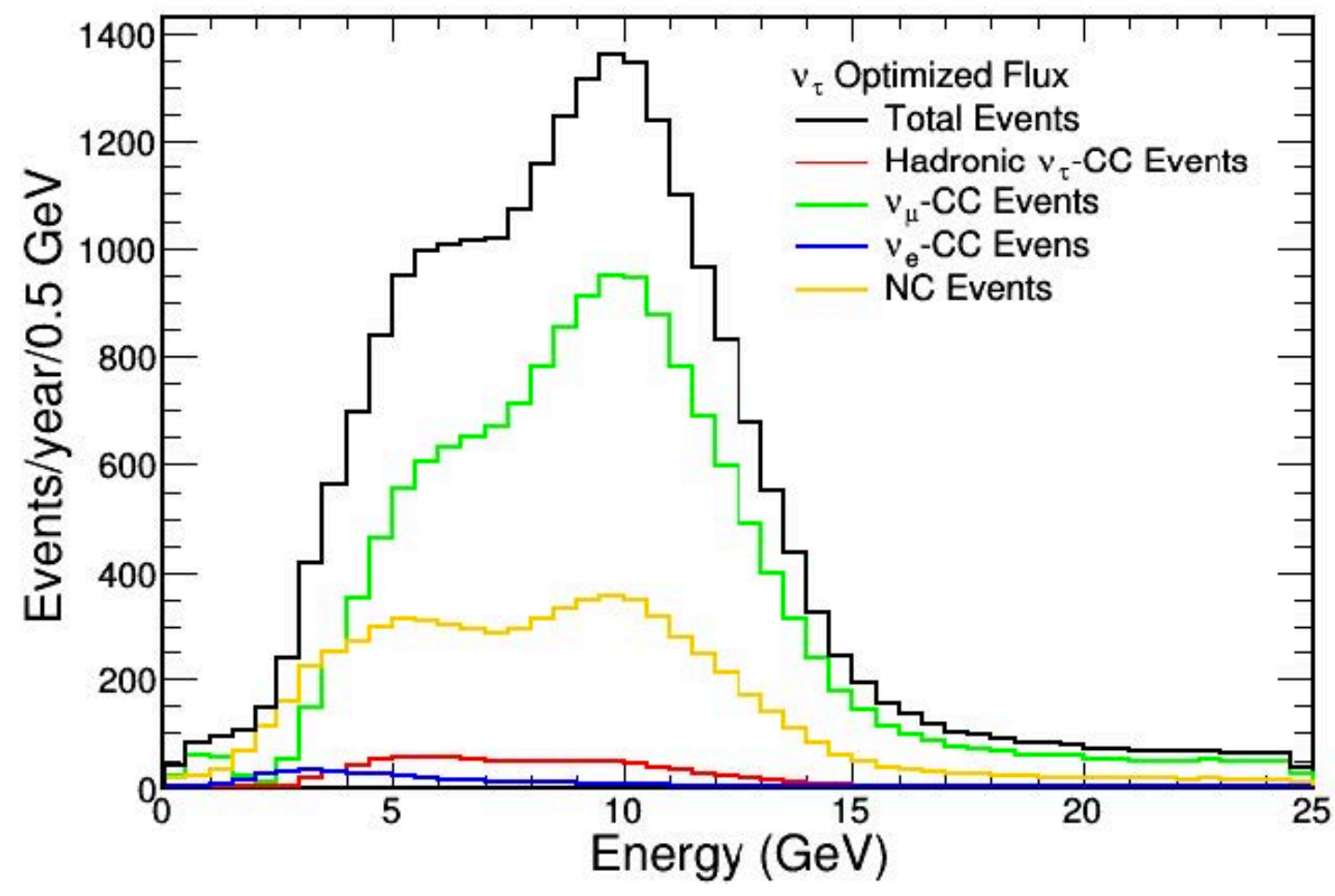




\section{Conclusions and outlook}

- The DUNE Near Detector Design Group (NDDG) has been formed to deliver a CDR for near detectors \& the facility

- I have outlined the basic approach that is being studied and which will form the bases of the CDR to a large extent

- Powerful, high-precision, full capability (calorimetric, spectrometer, PID, multiple target nuclei, off-axis measurements) detector systems

- LAr, MPD (HPgTPC+ECAL+Magnet+ $\mu$ tagger), 3DST

- With these detectors and the LBNF beam we will accumulate enormous statistics in all channels, including neutrino-electron elastic scattering.

- 1.5M v $v_{\mu} \mathrm{CC}$ events/yr-ton (FHC)

- Aggressive 3-pronged approach to CPV

- Opportunities to study physics beyond the $v S M$ are extensive 


\section{THANK YOU}

\section{Fermilab}

50 Years of Discovery 